Brian S. Thomson, Mathematics Department, Simon Fraser University,

B. C., Canada V5A 1S6; e-mail: thomson@cs.sfu.ca

\title{
SOME PROPERTIES OF VARIATIONAL MEASURES
}

\author{
Dedicated to the memory of Vasile Ene (1957-1998).
}

\begin{abstract}
Recently several authors have established a remarkable property of the variational measures associated with a function. Expressed in classical language, this property asserts that if a function is $\mathrm{ACG}_{*}$ on all sets of Lebesgue measure zero then the function must be globally $\mathrm{ACG}_{*}$. This article is an exposition of some ideas related to this property with the intention of bringing it to the attention of a wider audience than these original papers might attract.
\end{abstract}

Recent years have seen continued interest in the variational measures associated with a function, e.g., [1], [2], [3], [4], [7], [8], [9], [12], [13], [14], [15], [17], [18], [20], and [21].

In the simplest setting a function $f:[a, b] \rightarrow \mathbb{R}$ is given and one constructs a measure $\mu_{f}$ that carries the variational information about $f$. If $f$ is of bounded variation then $\mu_{f}$ is the usual Lebesgue-Stieltjes measure associated with the total variation function of $f$. In general a measure $\mu_{f}$ can be constructed for arbitrary functions and which has considerable power to express properties of $f$. Perhaps the nicest elementary uses of this measure would be in the following assertions.

If $f:[a, b] \rightarrow \mathbb{R}$ then a necessary and sufficient condition for the identity $f(x)-f(a)=\int_{a}^{x} f^{\prime}(t) d t$ in the sense of the Lebesgue integral is that $\mu_{f}$ is finite and absolutely continuous with respect to Lebesgue measure on $[a, b]$.

If $f:[a, b] \rightarrow \mathbb{R}$ then a necessary and sufficient condition for the identity $f(x)-f(a)=\int_{a}^{x} f^{\prime}(t) d t$ in the sense of the Denjoy-Perron integral is that $\mu_{f}$ is $\sigma$-finite and absolutely continuous with respect to Lebesgue measure on $[a, b]$.

Mathematical Reviews subject classification: 26A45, 26A39, 28A12

Received by the editors August 20, 1998 
W. F. Pfeffer, with characteristic insight, conjectured in 1994 that in the latter assertion the assumption that $\mu_{f}$ is $\sigma$-finite may be dropped, in fact that the property that $\mu_{f}$ is absolutely continuous with respect to Lebesgue measure on $[a, b]$ is already enough to deduce that it is also $\sigma$-finite. This remarkable property of the variational measure has since been proved, both on the real line ([2], [3], [13]) and in various higher dimensional versions ([4], [7], [8]). It is this property that we propose to study in this short article.

The property can be expressed directly, too, in the more classical language familiar to most real analysts. Roughly it asserts that to test that a function is $\mathrm{ACG}_{*}$ on a set $E$ it would be enough to test that it is $\mathrm{ACG}_{*}$ on measure zero subsets of $E$ (cf. Ene [12, p. 58]). To better appreciate the surprising feature of this observation we should note that it was entirely overlooked by Denjoy and Saks who, most of us surely felt, had exhausted the study of the $\mathrm{VBG}_{*}$ and $\mathrm{ACG}_{*}$ classes of functions. Since the proof does not require techniques with which they were unfamiliar it was only that this property did not occur to them.

It is, by no means, the case that all Borel measures on the interval $[a, b]$ would have the Pfeffer property. For example, simply take $\mu(B)=0$ for all Borel sets of measure zero and $\mu(B)=\infty$ for the remaining Borel sets.

What is there about the variational measures that allows this feature, that the behavior on the measure zero Borel sets imposes some global behavior? Since the proof in [2] uses the language of $\mathrm{ACG}_{*}$ functions and that in [14] uses the language of $\mathrm{VBG}_{*}$ functions it may not be immediately clear that this is a feature of the method used to construct the measures and not a property merely of functions. The measure arguments in [4], [8] and [9] require different techniques since they address the problem in higher dimensions. The simple technique used here is adapted from [3].

§1. Let us begin by recalling the method (sometimes known as Method III) defining the measure associated with any nonnegative interval function $\tau$ on $[a, b]$. Let $E \subset[a, b]$, let $\delta$ be a gauge on $E$ (i.e., $\delta$ is a positive function defined on $E$ ) and write

$$
V(\tau, E, \delta)=\sup \left\{\sum \tau\left(a_{i}, b_{i}\right)\right\},
$$

where the supremum is taken over all disjoint collections $\left\{\left(a_{i}, b_{i}\right)\right\}$ of open subintervals of $(a, b)$ for which there is a point $\xi_{i} \in E \cap\left(a_{i}, b_{i}\right)$ satisfying $b_{i}-a_{i}<\delta\left(\xi_{i}\right)$. Then write

$$
\mu_{\tau}^{*}(E)=\inf \{V(\tau, E, \delta): \delta \text { is a gauge on } E\} .
$$

It can be verified that $\mu_{\tau}^{*}$ is a metric outer measure on $[a, b]$. Since it is a metric outer measure its restriction to the Borel sets is a measure $\mu_{\tau}$; we 
call $\mu_{\tau}$ the Method III measure associated with the interval function $\tau$. If $f$ is continuous and monotonic and $\tau(a, b)=|f(b)-f(a)|$ then $\mu_{\tau}$ is precisely the Lebesgue-Stieltjes measure generated by $f$. If $f$ is continuous and has bounded variation then $\mu_{\tau}$ is the Lebesgue-Stieltjes measure associated with the total variation function for $f$. (Accounts of metric outer measures can be found in numerous texts, for example in Bruckner et al. [6] and Edgar [10] or [11] where also this method of construction is discussed.)

We show that all measures constructed in this manner have the Pfeffer property, in fact that if $\mu_{\tau}(B)$ is $\sigma$-finite for all Lebesgue measure zero closed sets $F \subset E$ where $E$ is closed then $\mu_{\tau}$ is $\sigma$-finite on $E$. In particular it follows that if $\mu_{\tau}$ vanishes on all Lebesgue measure zero subsets of $E$ then $\mu_{\tau}$ is $\sigma-$ finite on $E$. The method of proof uses a standard Baire category argument and a clever construction of a measure zero Cantor set in $E$; it is adapted from [2] where it is used in the setting of $\mathrm{ACG}_{*}$ functions (cf. also Pfeffer [17, pp. 7-8]).

Theorem 1. Let $\mu_{\tau}$ be a measure constructed from an interval function $\tau$ and let $E \subset[a, b]$ be closed. If $\mu_{\tau}$ is $\sigma$-finite on all closed subsets of $E$ that have zero Lebesgue measure, then $\mu_{\tau}$ is $\sigma$-finite on $E$.

Proof. Let $P$ be the set of all points $x \in E$ for which $\mu_{\tau}$ is non $\sigma$-finite on $E \cap(c, d)$ for every interval $(c, d)$ containing $x$. We claim that $P$ is empty. If so the theorem is easy to prove. Associated with every point $x$ in the compact set $E$ is an open interval $I_{x}$ so that $\mu_{\tau}$ is $\sigma$-finite on $E \cap I_{x}$. A compactness argument reduces this open cover to a finite subcover and shows that $\nu_{\tau}$ is $\sigma$-finite on $E$.

Let us show that $P$ is empty by obtaining a contradiction from the assumption that $P \neq \emptyset$.

If $P$ is nonempty then it is a perfect subset of $[a, b]$. Clearly $P$ is closed. We claim that it has no isolated points. To see this suppose that $x_{0}$ is, if possible, an isolated point of $P$. Then, since $\left\{x_{0}\right\} \cap E$ is measure zero trivially, $\mu_{\tau}$ is $\sigma$-finite on $\left\{x_{0}\right\} \cap E$ (i.e., it is finite). Because $x_{0}$ is an isolated point of $P$ it follows that $\mu_{\tau}$ is also $\sigma$-finite on $\left[a_{i}, a_{i+1}\right] \cap E$ for some sequence of points $a_{i} \nearrow a$ and it is $\sigma$-finite on $\left[b_{i+1}, b_{i}\right] \cap E$ for some sequence of points $b_{i} \searrow a$. It follows, then that $\mu_{\tau}$ is $\sigma$-finite on $\left[a_{1}, b_{1}\right] \cap E$ which means that $x_{0}$ could not have been a point of $P$.

Continuing to assume that $P \neq \emptyset$, we choose a finite, disjoint collection

$$
I_{11}, I_{12}, I_{13}, \ldots
$$

of open subintervals of $(a, b)$ (at least three such intervals in any case) so that 
each contains a point of $P$ and so that

$$
\sum_{j}\left|I_{1 j}\right|<1 / 2
$$

(here $|I|$ is used to denote the length of an interval $I$ ) and

$$
\sum_{j} \tau\left(I_{1 j}\right)>2
$$

The reason we can do this is that if $J$ is any interval containing a point of $P$ then $V(\tau, P \cap J, \delta)=\infty$ for any $\delta$. For if not then $\mu_{\tau}(P \cap J)<\infty$ and $\mu_{\tau}$ is $\sigma$-finite on each set $E \cap\left(c_{i}, d_{i}\right)$ where $\left\{\left(c_{i}, d_{i}\right)\right\}$ is the sequence of intervals complementary to $P$ in $J$. But

$$
E \cap J=(P \cap J) \cup \bigcup_{i}\left(E \cap\left(c_{i}, d_{i}\right)\right)
$$

and it would follow that $\mu_{\tau}$ is $\sigma$-finite on $E \cap J$ which is not possible if $J$ contains a point of $P$.

Since $V(\tau, P \cap J, \delta)=\infty$ for any $\delta$, a disjoint sequence of subintervals $I_{1}$, $I_{2}, I_{3}, \ldots$ of $J$ each containing a point of $P$ can be selected with

$$
\sum_{k} \tau\left(I_{k}\right)
$$

as large as we please.

Thus, since $P$ is perfect we can begin by selecting three disjoint intervals $J, J^{\prime}$, and $J^{\prime \prime}$ each containing a point of $P$, and each with length less than $1 / 6$, and find inside them enough further open subintervals to provide the sequence $I_{11}, I_{12}, I_{13}, \ldots$ containing at least three members and with the desired properties. Now inside each interval $I_{11}, I_{12}, I_{13}, \ldots$ we can apply the same argument to find still smaller intervals.

Let us set $I_{01}=(a, b)$ and proceed inductively using the same argument at each stage. We construct disjoint intervals

$$
I_{i 1}, I_{i 2}, I_{i 3}, \ldots
$$

so that

a. each $I_{i j}$ is an open subinterval of some previous level interval $I_{(i-1) k}$,

b. each interval contains a point of $P$, 
c. each such interval $I_{(i-1) k}$ contains at the next level at least three such intervals $I_{i j}$ and

d. for any $i=1,2,3, \ldots$

$$
\sum_{j}\left|I_{i j}\right|<2^{-i}
$$

and, finally

e. for any $i=1,2,3, \ldots$ and if there is an interval $I_{(i-1) k}$ then

$$
\sum_{j}\left\{\tau\left(I_{i j}\right): I_{i j} \subset I_{(i-1) k}\right\}>2^{i} .
$$

Define now the set

$$
N=E \cap \bigcap_{i} \bigcup_{j} \overline{I_{i j}}
$$

This set $N$ is closed. Compactness of the sets ensures that it is nonempty. It is of Lebesgue measure zero because of the requirement (e) in the construction of the intervals.

Since $N$ is a closed, measure zero subset of $E$ the measure $\mu_{\tau}$ must be, by hypothesis, $\sigma$-finite on $N$. Let $N_{1}, N_{2}, N_{3}$, be a sequence of disjoint Borel subsets of $N$ on each of which $\mu_{\tau}$ is finite and whose union is all of $N$. Choose a gauge $\delta$ on $N$ so that

$$
V\left(\tau, N_{p}, \delta\right)<\infty
$$

for each $p=1,2,3, \ldots$ Let

$$
E_{m}=\{x \in N: \delta(x)>1 / m\}
$$

for each $m=1,2,3, \ldots$ Note that $E_{m} \nearrow N$. Thus the sets $\left\{E_{m} \cap N_{p}\right\}$ for $m=1,2,3, \ldots$ and $p=1,2,3, \ldots$ form a countable cover of $N$. By the Baire category theorem there is an open interval $I$ and a member $N_{p} \cap E_{m}$ of the cover so that $N_{p} \cap E_{m}$ is dense in the nonempty portion $N \cap I$. By passing to a subinterval if necessary, we can assume that $|I|<1 / \mathrm{m}$.

On the one hand, we have from the way in which we constructed the gauge

$$
V\left(\tau, E_{m} \cap N_{p}, \delta\right) \leq V\left(\tau, N_{p}, \delta\right)<\infty .
$$

But, on the other hand, since $I$ contains points of $N$ there must be for all sufficiently large $i$ some $k$ so that $I_{(i-1) k} \subset I$. Each interval

$$
I_{i j} \subset I_{(i-1) k}
$$


must contain a point of $N$; since $N_{p} \cap E_{m}$ is dense in the portion $N \cap I$ each such interval also contains a point $\xi$ of $N_{p} \cap E_{m}$. But the length of such an interval would be smaller than $I$ which is smaller than $1 / m$ which is smaller than $\delta(\xi)$. Consequently from the requirement (e)

$$
2^{i}<\sum_{j}\left\{\tau\left(I_{i j}\right): I_{i j} \subset I_{(i-1) k}\right\} \leq V\left(\tau, E_{m} \cap N_{p}, \delta\right) .
$$

This would be valid for all sufficiently large $i$ and that is impossible because of (1). Thus we have reached a contradiction and completed the proof.

To express our theorem in another way we could observe that if $\mu_{\tau}$ is non $\sigma$-finite on a closed set $E$ then $\mu_{\tau}$ is non $\sigma$-finite on many closed null subsets of $E$. How many? Can one say anything about the subsets of $E$ of Hausdorff dimension smaller than 1? For answers to these questions (and others) in $\mathbb{R}^{n}$ for any dimension $n$ see the interesting papers of B. Bongiorno et al. [4] and Z. Buczolich and W. F. Pfeffer [7].

$\S 2$. We can take another perspective on the result in Section 1. Implicit in this method of constructing a measure is a differentiation basis and the derivates of the interval function $\tau$ play an important role in studying the measure $\mu_{\tau}$. (For a deeper account of this role see [20].) be

Define for any $x \in(a, b)$, the upper derivate of $\tau$ at the point $x, \bar{D} \tau(x)$, to

$$
\inf _{\delta>0} \sup \left\{\frac{\tau(I)}{|I|}: \quad I \text { an open subinterval of }(a, b) \text { with } x \in I \text { and }|I|<\delta\right\} .
$$

The lemma shows that the $\sigma$-finiteness of the measure $\mu_{\tau}$, which was our concern in the preceding section, has a great deal to do with this derivate.

Lemma. Let $\tau$ be an arbitrary nonnegative interval function and $\mu_{\tau}$ the measure generated by it, let $E$ be a Borel subset of the interval $[a, b]$, and write

$$
E_{\infty}=\{x \in E: \bar{D} \tau(x)=\infty\} .
$$

Then $E_{\infty}$ is a Borel subset of $E$ and $\mu_{\tau}$ is $\sigma$-finite on $E \backslash E_{\infty}$. If, moreover, $\mu_{\tau}$ is $\sigma$-finite on $E$ then $E_{\infty}$ has Lebesgue measure zero.

Proof. The set $E \backslash E_{\infty}$ is the union of the sequence of sets

$$
E_{n}=\{x \in E: \bar{D} \tau(x)<n\}
$$


As easy estimate shows that $\mu_{\tau}\left(E_{n}\right) \leq n(b-a)$ and this shows that $\mu_{\tau}$ is $\sigma$-finite on $E \backslash E_{\infty}$. Standard arguments suffice to show that all sets here are Borel (cf. $[20, \S 4.2]$ ).

Suppose now that $K$ is any closed subset of $E_{\infty}$ for which $\mu_{\tau}(K)<\infty$ and let $c>0$. We may choose a gauge $\delta$ on $K$ so that

$$
V(\tau, K, \delta) \leq \mu_{\tau}(K)+1
$$

The collection $\mathcal{C}$ of all open subintervals $I$ of $(a, b)$ with the property that $\tau(I)>c|I|$ and, for some $x \in I,|I|<\delta(x)$ is a Vitali cover of $K$. Thus there must exist a disjoint sequence $\left\{I_{i}\right\} \subset \mathcal{C}$ so that

$$
|K| \leq \sum_{i}\left|I_{i}\right|
$$

(here $|K|$ denotes the Lebesgue measure of the set $K$ ). This gives us that

$$
c|K| \leq \sum_{i} c\left|I_{i}\right| \leq \sum_{i} \tau\left(I_{i}\right) \leq V(\tau, K, \delta) \leq \mu_{\tau}(K)+1 .
$$

The inequality

$$
c|K| \leq \mu_{\tau}(K)+1
$$

can be valid for all $c>0$ only if $|K|=0$. Thus there can be no closed subsets of $E_{\infty}$ of positive Lebesgue measure that have finite $\mu_{\tau}$ measure. This proves the second assertion of the lemma.

Using this lemma and Theorem 1 we can prove the following theorem asserting a very weak condition under which a.e. finiteness of the derivate $\bar{D} \tau(x)$ can be concluded. Prior to the conjecture of Pfeffer this condition might have seemed impossibly weak. Higher dimensional variants of this theorem may be found in B. Bongiorno et al. [4] and Buczolich and Pfeffer [9].

Theorem 2. Let $\mu_{\tau}$ be a measure constructed from an interval function $\tau$ and let $E \subset[a, b]$ be Lebesgue measurable. If $\mu_{\tau}$ is $\sigma$-finite on all closed subsets of $E$ that have zero Lebesgue measure, then the set

$$
E_{\infty}=\{x \in E: \bar{D} \tau(x)=\infty\}
$$

has Lebesgue measure zero.

Proof. The set $E_{\infty}$ is measurable if $E$ is and so, to prove that it has Lebesgue measure zero, it is enough to show that every closed subset has Lebesgue measure zero. This follows immediately from Theorem 1 and the Lemma. 


\section{References}

[1] B. Bongiorno, W. F. Pfeffer, and B. S. Thomson, A full descriptive definition of the gage integral, Canadian Math. Bull., 39(4) (1996), 390-401.

[2] B. Bongiorno, L. Di Piazza and V. Skvortsov, The essential variation of a function and some convergence theorems, Anal. Math. (1) 22 (1996), $3-12$.

[3] B. Bongiorno, L. Di Piazza and V. Skvortsov, A new full descriptive characterization of the Denjoy-Perron integral, Real Anal. Exch., 2 (1995/96), No. 2, 656-663.

[4] B. Bongiorno, L. Di Piazza and D. Preiss, Infinite variation and derivatives in $\mathbb{R}^{n}$, Journal of Math. Anal. Appl., 224 (1998) no. 1, 22-33.

[5] A. M. Bruckner, Differentiation of Real Functions, Springer-Verlag (1978).

[6] A. M. Bruckner, J. B. Bruckner and B. S. Thomson, Real Analysis Prentice-Hall (1996).

[7] Z. Buczolich and W. F. Pfeffer, When absolutely continuous implies $\sigma$ finite, Bull. Csi., Acad. Royale Belgique, serie 6, 1-6 (1997), 155-160.

[8] Z. Buczolich and W. F. Pfeffer, Variations of additive functions, Czech. Math. J., 47 (122) (1997), no. 3, 525-555.

[9] Z. Buczolich and W. F. Pfeffer, On absolute continuity, Journal of Math. Anal. Appl., 222, (1998) no. 1, 64-78.

[10] G. A. Edgar, Measure, Topology, and Fractal Geometry, Springer-Verlag, New York (1990).

[11] G. A. Edgar, Integral, Probability, and Fractal Measures, Springer-Verlag, New York (1998).

[12] V. Ene, Real functions - current topics, Lect. Notes in Math., vol. 1603, Springer-Verlag, 1995.

[13] V. Ene, Characterizations of $V B^{*} G \cap(N)$, Real Anal. Exch., 23 (1997/98) no. 2, 571-599.

[14] V. Ene, Thomson's variational measure, Real Anal. Exch., 24 (1998/99) no. 2 . 
[15] W. F. Pfeffer, The Riemann Approach to Integration: Local Geometric Theory. Cambridge University Press (1993).

[16] W. F. Pfeffer, The generalized Riemann-Stieltjes integral, Real Anal. Exch., 21, No. 2, 521-547.

[17] W. F. Pfeffer, On variations of functions of one real variable, Comment. Math.,Univ. Carolin., 38, no. 1(1997), 61-71.

[18] W. F. Pfeffer and B.S. Thomson, Measures defined by gages, Canad. Journal of Math. 44 (6) 1992, 1303-1316.

[19] S. Saks, Theory of the Integral, Dover, (1937).

[20] B. S. Thomson, Derivates of Interval Functions, Memoir American Math. Soc., 452, Providence, 1991.

[21] B. S. Thomson, $\sigma$-finite Borel measures on the real line. Real Anal. Exch., 23, (1997-98) no. 1, 185-192. 